VoL. 63 (2001) [257-268]

\title{
ALTERNATIVE QUASIALGEBRAS
}

\author{
Helena Albuquerque, Alberto Elduque \\ and José María PÉrez-IzQuierdo
}

\begin{abstract}
Alternative division quasialgebras which are not associative, are characterised. Their grading groups are shown to be always Abelian and they are built from some specific graded associative algebras by means of a graded Cayley-Dickson process.
\end{abstract}

\section{INTRODUCTION}

Given a group $G$, a field $k$ and a cocycle $\phi: G \times G \times G \rightarrow k^{\times}$(that is, $\phi(a, e, b)=1$ for any $a, b \in G$, where $e$ denotes the neutral element, and

$$
\phi(a, b, c) \phi(a, b c, d) \phi(b, c, d)=\phi(a b, c, d) \phi(a, b, c d)
$$
for any $a, b, c, d \in G), G$-graded $k$-algebras $A=\bigoplus_{g \in G} A_{g}$ satisfying the "twisted" asso-
ciative law

$$
\left(x_{a} x_{b}\right) x_{c}=\phi(a, b, c) x_{a}\left(x_{b} x_{c}\right)
$$

for any homogeneous elements $x_{a} \in A_{a}, x_{b} \in A_{b}, x_{c} \in A_{c}$, were introduced in [2] and called $G$-graded quasialgebras.

For some groups, these have been studied in $[1,3]$.

If the cocycle is trivial, a quasialgebra is nothing else but a graded associative algebra. An interesting feature is that some noteworthy nonassociative algebras, such as Cayley-Dickson algebras, are quasialgebras too, for suitable groups and cocycles.

Let, $F: G \times G \rightarrow k^{\times}$be a 2-cochain (that is, a map satisfying $F(e, a)=1=F(a, e)$ for any $a \in G$ ). Then the group algebra $k G$ "twisted" by considering the new product on it determined by

$$
x \cdot_{F} y=F(x, y) x y
$$

for any $x, y \in G$, is a $G$-graded quasialgebra, denoted by $k_{F} G$, whose cocycle is $\partial F$. Hence $k_{F} G$ is associative if and only if $F$ is a 2 -cocycle. (These algebras have been

Received 30th May, 2000

The first author was supported by CMUC-FCT and by Praxis PCEX/P/MAT/114/96, the second author by the Spanish DGES (Pb 97-1291-C03-03), and the third author by DGES (Pb 97-1291-C0302) and by the Universidad de La Rioja (API 98/BI5, API 99/B04 and API 00/B04).

Copyright Clearance Centre, Inc. Serial-fee code: 0004-9727/01 . \$A2.00+0.00. 
considered in [6].) Any Cayley-Dickson algebra over a field $k$ of characteristic $\neq 2$ is a quasialgebra $k_{F} G$, where $G=\mathbb{Z}_{2} \times \mathbb{Z}_{2} \times \mathbb{Z}_{2}$, for a suitable cochain $F$ (see [2]).

A quasialgebra $A=\bigoplus_{g \in G} A_{g}$ is said to be a division quasialgebra if $A_{g} \neq 0$ for any $g \in G$ and for any $g, h \in G$ and $0 \neq x_{g} \in A_{g}$, the linear maps

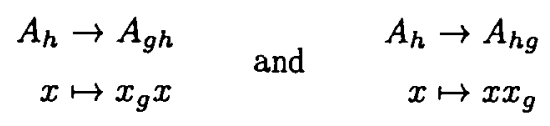

are bijective. Associative division quasialgebras are just associative graded division algebras, which are building blocks in the theory of graded associative rings (see [5]). Quasialgebras, $k_{F} G$ and, in particular, Cayley-Dickson algebras over fields of characteristic not equal to 2, are examples of division quasialgebras too.

The purpose of this paper is to characterise those division quasialgebras which are alternative but not associative. It will be proven that if $A=\bigoplus_{g \in G} A_{g}$ is such a division quasialgebra, then the grading group $G$ must be Abelian and contain a subgroup $N$ with quotient $G / N$ isomorphic to $\mathbb{Z}_{2} \times \mathbb{Z}_{2} \times \mathbb{Z}_{2}$. Moreover, the subalgebra $A_{N}=\bigoplus_{g \in N} A_{g}$ is then a graded associative division algebra, of a very specific nature, and $A$ is built from $A_{N}$ by means of a "graded version" of the Cayley-Dickson doubling process.

Alternative $\mathbb{Z}^{n}$-graded algebras $A=\bigoplus_{\alpha \in \mathbb{Z}^{n}} A_{\alpha}$ over a field $k$ of characteristic not equal to 2 with $\operatorname{dim}_{k} A_{\alpha}=1$ and $A_{\alpha} A_{\beta}=A_{\alpha+\beta}$ for any $\alpha, \beta \in \mathbb{Z}^{n}$, called alternative tori, have been considered in [4] in connection with some extended affine Lie algebras. It is clear that such algebras are twisted group algebras and hence they provide noteworthy examples of alternative division quasialgebras. The characterisation of these algebras given in [4, Theorem 1.25] is subsumed in our main result (Theorem 3.12).

\section{PRELiminaries}

Let $A$ be an algebra over a field $k$ and let $[x, y]=x y-y x$ and $(x, y, z)=$ $(x y) z-x(y z)$ be the commutator and associator of elements $x, y, z \in A$. The nucleus $N(A)$, commutative centre $K(A)$ and centre $Z(A)$ are defined by (see $[8$, p. 136]):

$$
\begin{aligned}
N(A) & =\{x \in A:(x, A, A)=(A, x, A)=(A, A, x)=0\}, \\
K(A) & =\{x \in A:[x, A]=0\} \\
Z(A) & =N(A) \cap K(A) .
\end{aligned}
$$

The sets $N(A)$ and $Z(A)$ are always subalgebras of $A$ and, if the algebra $A$ is alternative (that is, $x^{2} y=x(x y)$ and $y x^{2}=(y x) x$ for any $x, y \in A$ ), then $K(A)$ is also a subalgebra of $A$ and, moreover, $3 K(A) \subseteq Z(A)$. 
Given a quasialgebra $A=\bigoplus_{g \in G} A_{g}$, with associated cocycle $\phi$, the set

$$
N(G, \phi)=\{g \in G: \phi(g, G, G)=\phi(G, g, G)=\phi(G, G, g)=1\}
$$

will be called the nucleus of the pair $(G, \phi)$.

LEMMA 2.1. $N(G, \phi)$ is a subgroup of $G$, and for any $g \in N(G, \phi)$ and $a, b, c \in G$ :

$$
\begin{gathered}
\phi(a, b, c)=\phi(a, b, c g)=\phi(g a, b, c) \\
\phi(a g, b, c)=\phi(a, g b, c) \quad \text { and } \phi(a, b g, c)=\phi(a, b, g c)
\end{gathered}
$$

Proof: For $g, h \in N(G, \phi)$ and $a, b \in G$

$$
\begin{aligned}
1 & =\phi(g, h, a) \phi(g, h a, b) \phi(h, a, b) \\
& =\phi(g h, a, b) \phi(g, h, a b)=\phi(g h, a, b),
\end{aligned}
$$

so $\phi(g h, G, G)=1$ and, similarly, $\phi(G, g h, G)=\phi(G, G, g h)=1$ and $g h \in N(G, \phi)$. On the other hand, since $\phi(e, G, G)=1$,

$$
\begin{aligned}
\phi\left(g^{-1}, a, b\right) & =\phi\left(g, g^{-1}, a\right) \phi\left(g, g^{-1} a, b\right) \phi\left(g^{-1}, a, b\right) \\
& =\phi(e, a, b) \phi\left(g, g^{-1}, a b\right)=1
\end{aligned}
$$

so $\phi\left(g^{-1}, G, G\right)=1$ and, in the same way, $\phi\left(G, G, g^{-1}\right)=1$. Also

$$
\begin{aligned}
1 & =\phi\left(a, g, g^{-1}\right) \phi(a, e, b) \phi\left(g, g^{-1}, b\right) \\
& =\phi\left(a g, g^{-1}, b\right) \phi\left(a, g, g^{-1} b\right)=\phi\left(a g, g^{-1}, b\right),
\end{aligned}
$$

so $\phi\left(G, g^{-1}, G\right)=\phi\left(G g, g^{-1}, G\right)=1$ too and $g^{-1} \in N(G, \phi)$.

Now, simple computations using the cocycle condition give the result. related:

For division quasialgebras $A=\bigoplus_{g \in G} A_{g}$, the nuclei $N(G, \phi)$ and $N(A)$ are closely

LEMMA 2.2. Let $A=\bigoplus_{g \in G} A_{g}$ be a division quasialgebra with cocycle $\phi$, then $N(A)=\bigoplus_{g \in N(G, \phi)} A_{g}$.

Proof: It is clear from the definitions that $N(A)$ is a graded subalgebra of $A$ and that $\bigoplus_{g \in N(G, \phi)} A_{g} \subseteq N(A)$. Also, if $0 \neq x_{g} \in A_{g} \cap N(A)$, then by the division property, $A_{g}=A_{e} x_{g} \subseteq N(A)$, since $N(A)$ is a subalgebra. For any $a, b \in G$ and $0 \neq x_{a} \in A_{a}$, $0 \neq x_{b} \in A_{b}$ :

$$
0=\left(x_{g}, x_{a}, x_{b}\right)=\left(x_{g} x_{a}\right) x_{b}-x_{g}\left(x_{a} x_{b}\right)=(\phi(g, a, b)-1) x_{g}\left(x_{a} x_{b}\right),
$$


so that $\phi(g, G, G)=1$ since $x_{g}\left(x_{a} x_{b}\right) \neq 0$. In the same way $\phi(G, g, G)=1=$ $\phi(G, G, g)$. Therefore, $g \in N(G, \phi)$ and $N(A) \subseteq \underset{g \in N(G, \phi)}{\bigoplus} A_{g}$.

Notice that if $A$ is an alternative algebra, then the associator is a skew-symmetric map on its arguments, hence $N(A)=\{x \in A:(x, A, A)=0\}$. Then, if $A=\bigoplus_{g \in G} A_{g}$ is an alternative division quasialgebra and $g \in G, \phi(g, G, G)=1$ if and only if $\left(A_{g}, A, A\right)=0$, and this last condition is equivalent to $\left(A, A_{g}, A\right)=0$ and $\left(A, A, A_{g}\right)=0$. Thus, the conditions $\phi(g, G, G)=1, \phi(G, g, G)=1$ and $\phi(G, G, g)=1$ are equivalent.

In what follows, alternative algebras which are not associative will be said to be strictly alternative.

\section{Alternative division Quasialgebras}

The first thing to do, in order to characterise the strictly alternative division quasialgebras, is to show that the grading group has to be Abelian.

Lemma 3.1. Let $A=\bigoplus_{g \in G} A_{g}$ be a strictly alternative division quasialgebra. Then, for any elements $a, b, c \in G$ and $x_{a} \in A_{a}, x_{b} \in A_{b}$ :

$$
(\phi(c, a, b)-1) x_{a} x_{b}=-(\phi(c, b, a)-1) x_{b} x_{a} .
$$

Proof: Take $0 \neq x_{c} \in A_{c}$. By the skew-symmetry of the associator, $\left(x_{c}, x_{a}, x_{b}\right)=$ $-\left(x_{c}, x_{b}, x_{a}\right)$ and, since $\left(x_{c}, x_{a}, x_{b}\right)=(\phi(c, a, b)-1) x_{c}\left(x_{a} x_{b}\right)$,

$$
(\phi(c, a, b)-1) x_{c}\left(x_{a} x_{b}\right)=-(\phi(c, b, a)-1) x_{c}\left(x_{b} x_{a}\right) .
$$

The division property implies the result.

THEOREM 3.2. Let $A=\bigoplus_{g \in G} A_{g}$ be a strictly alternative division quasialgebra. Then $G$ is $A$ belian and $N(A)=Z(A)$.

Proof: By [8, Exercise 6, p. 144], $(A, A, z)[z, N(A)]=0$ for any $z \in A$, and hence, by linearisation, $(A, A, z)[N(A), N(A)]=0$, so $(A, A, A)[N(A), N(A)]=0$. Since $A$ is a strictly alternative division quasialgebra, this forces $[N(A), N(A)]=0$ and also $\left[A_{a}, N(A)\right]=0$ for any $a \notin N(G, \phi)$. Therefore $[A, N(A)]=0$ and $N(A)=$ $Z(A)$. Thus, for any $g \in N(G, \phi), a \in G$ and $0 \neq x_{g} \in A_{g}, 0 \neq x_{a} \in A_{a}$,

$$
x_{g} x_{a}=x_{a} x_{g} \in A_{a g} \cap A_{g a}
$$

and hence $g a=a g$ and $N(G, \phi) \subseteq Z(G)$. 
Moreover, if $a, b \in G$ satisfy $\phi(G, a, b) \neq 1$, then by Lemma 3.1, for any $0 \neq x_{a} \in$ $A_{a}$ and $0 \neq x_{b} \in A_{b}$,

$$
0 \neq x_{a} x_{b} \in A_{a b} \cap A_{b a}
$$

and hence $a b=b a$. On the other hand, if $\phi(G, a, b)=1$, then $\left(A, A_{a}, A_{b}\right)=0$ and, by [8, Lemma 3 , p. 134], $\left[A_{a}, A_{b}\right] \subseteq N(A)$, which implies, since $N(A)$ is a graded subalgebra, that either $a b=b a$ or $a b, b a \in N(G, \phi) \subseteq Z(G)$. In the latter case, $b a=b(a b) b^{-1}=(a b) b b^{-1}=a b$ since $a b \in Z(G)$. Therefore $G$ is Abelian, and the proof is complete.

Corollary 3.3. Let $A=\bigoplus_{g \in G} A_{g}$ be a strictly alternative division quasialgebra. Then $K=A_{e}$ is a field and $A$ is a unital $K$-algebra $\left(1 \in A_{e}\right)$.

Proof: By Theorem 3.2, $K=A_{e}$ is an associative and commutative division algebra, so it is a field, and it is contained in the centre of $A$. Moreover, if 1 denotes the unit element of $K$, the division property and the conditions $\left(A_{e}, A_{e}, A\right)=0=$ $\left(A, A_{e}, A_{e}\right)$ imply that 1 is the unit element of $A$.

Lemma 2.1 and the commutativity of $G$ immediately imply the following consequence, where $N(G, \phi)$ is denoted by $N$ for short:

Corollary 3.4. Let $A=\bigoplus_{g \in G} A_{g}$ be a strictly alternative division quasialgebra. Then $\phi$ induces a cocycle $\widehat{\phi}: G / N \times G / N \times G / N \rightarrow k^{\times}$defined by

$$
\widehat{\phi}(a N, b N, c N)=\phi(a, b, c) .
$$

In the next propositions, it will always be assumed that $A=\bigoplus_{g \in G} A_{g}$ is a strictly alternative division quasialgebra, with associated cocycle $\phi$ and, as before, $N(G, \phi)$ will be denoted simply by $N$.

Proposition 3.5. For any $g \in G, g^{2} \in N$.

Proof: If $g \in N, g^{2} \in N$ is clear since $N$ is a subgroup by Lemma 2.1.

If $g \notin N$ and $\left[A_{g}, A\right] \neq 0$, then there exists an element $h \in G$ with $\left[A_{g}, A_{h}\right]$ $\neq 0$. Let $x_{g} \in A_{g}$ and $x_{h} \in A_{h}$ be such that $\left[x_{g}, x_{h}\right] \neq 0$. Then by [8, Corollary 2, p. 148] there are homogeneous elements $p, q, r \in N(A)$ with $p=\left[x_{g}, x_{h}\right]^{4}$ such that $p x_{g}^{2}-q x_{g}+r 1=0$. Multiplying by $p^{-1}$ we obtain $x_{g}^{2}-\bar{q} x_{g}+\bar{r} 1=0\left(x_{g}^{2} \neq 0\right)$ with $\bar{q}$ and $\bar{r}$ homogeneous elements in $N(A)$. Considering the homogeneous component in $A_{g^{2}}$, we have (since $g \notin N$ ) that $x_{g}^{2}+\bar{r} 1=0$, with $0 \neq \bar{r} \in A_{g^{2}} \cap N(A)$, and $g^{2} \in N$.

If $g \notin N$ but $\left[A_{g}, A\right]=0$, then necessarily the characteristic of $k$ is 3 by [8, Corollary 1, p. 136] and there are two possibilities: either $A$ is commutative, and then by $[8$, Lemma 8, p. 142$](A, A, A)^{2}=0$, which by the division property implies 
( $A, A, A)=0$, a contradiction to $A$ being not associative; or $A$ is not commutative, so there exist elements $a, b \in G, x_{a} \in A_{a}, x_{b} \in A_{b}$ with $\left[x_{a}, x_{b}\right] \neq 0$, which implies that $a^{2} \in N$ by the argument above. In this case, by [8, Equation (7) p. 136], $\left[x_{g} x_{a}, x_{b}\right]=x_{g}\left[x_{a}, x_{b}\right] \neq 0$ and $(g a)^{2} \in N$. Thus, $g^{2}=(g a)^{2}\left(a^{2}\right)^{-1} \in N$ (G is Abelian).

Proposition 3.6. For any elements $g, h \in G$ and $x_{g} \in A_{g}, x_{h} \in A_{h}$ :

(i) If $g \in N$ or $h \in N$ or $g h \in N$, then $\left[x_{g}, x_{h}\right]=0$.

(ii) If $\left[A_{g}, A_{h}\right] \neq 0$, then $x_{g} x_{h}+x_{h} x_{g}=0$.

Proof: For (i), if $g \in N, x_{g} \in N(A)=Z(A)$ (Theorem 3.2), so $\left[x_{g}, A\right]=0$. The same happens if $h \in N$. Now, if $g h \in N$ and $x_{g} \neq 0$, since $g^{2} \in N$, there exists an element $n \in N$ with $h=g n$. Then there is $x_{n} \in A_{n}$ such that $x_{h}=x_{g} x_{n}$, which implies that $\left[x_{g}, x_{h}\right]=\left[x_{g}, x_{g} x_{n}\right]$ and because the algebra generated by $x_{g}$ and $x_{n}$ is associative, the last expression is equal to $x_{g}\left[x_{g}, x_{n}\right]=0$ and so $\left[x_{g}, x_{h}\right]=0$.

For (ii), it can be assumed that $x_{g} \neq 0 \neq x_{h}$ and that $h \neq g$ because $g h \notin N$. If $\left[A_{g}, A_{h}\right] \neq 0$ (which implies that $g \notin N$ and $h \notin N$ ), since $A_{g}=A_{e} x_{g}$ and $A_{e} \subseteq N(A)$, then $\left[x_{g}, A_{h}\right] \neq 0$, and with the same argument $\left[x_{g}, x_{h}\right] \neq 0$. But then $0 \neq\left[x_{g}+x_{h}, x_{h}\right]=\left[x_{g}, x_{h}\right] \in A_{g h}$ and again, by [8, Corollary 2, p. 148], there are elements $p, q, r \in N(A)$ with $0 \neq p=\left[x_{g}+x_{h}, x_{h}\right]^{4} \in A_{g^{4} h^{4}}$ such that $p\left(x_{g}+x_{h}\right)^{2}-$ $q\left(x_{g}+x_{h}\right)+r 1=0$. The homogeneous component in $A_{(g h)^{5}}$ is

$$
p\left(x_{g} x_{h}+x_{h} x_{g}\right)-q_{(g h)^{4} g} x_{h}-q_{(g h)^{4} h} x_{g}+r_{(g h)^{5}} 1=0,
$$

where $q_{(g h)^{4} g}$ is the homogeneous component of $q$ in $A_{(g h)^{4} g}$ and similarly for $q_{(g h)^{4} h}$ and $r_{(g h)^{5}}$. But $(g h)^{4} \in N$ by Proposition 3.5 and $h \notin N$, which implies $(g h)^{4} h \notin N$ and $q_{(g h)^{4} h}=0$. Analogously $(g h)^{4} g \notin N,(g h)^{5} \notin N$ and we can conclude that $x_{g} x_{h}+x_{h} x_{g}=0$.

COROLLARY 3.7. There are no strictly alternative division quasialgebras over fields of characteristic 2 .

Proof: By Proposition 3.6, if the characteristic is equal to 2, then $[A, A]=$ 0 , so $A=3 A \subseteq N(A)$ by [8, Corollary 1, p. 136], a contradiction to $A$ not being associative.

Therefore, in what follows, the characteristic will be assumed to be not equal to 2 .

Proposition 3.8. The commutative centre of $A$ equals the nucleus $((A)=$ $N(A)=Z(A))$ and for any $g, h \in G \backslash N$ with $g h \notin N$ and $x_{g} \in A_{g}, x_{h} \in A_{h}$, $x_{g} x_{h}+x_{h} x_{g}=0$.

Proof: By extending scalars if necessary, the ground field $k$ may be assumed to be infinite. 
If the characteristic is not equal to 3 , then $K(A)=Z(A)(=N(A))$ by $[8$, Corollary 1, p. 136]. Then, since $g \in G \backslash N$ there is an element $a \in G$ and $x_{a} \in A_{a}$ such that $\left[x_{g}, x_{a}\right] \neq 0$ and hence there is a $0 \neq \alpha \in k$ such that $\left[x_{g}+\alpha x_{h}, x_{a}\right]^{4} \neq 0$. Using an already familiar argument, there are elements $p, q, r \in N(A)$, with $0 \neq p=$ $\left[x_{g}+\alpha x_{h}, x_{h}\right]^{4}$, such that

$$
p\left(x_{g}+\alpha x_{h}\right)^{2}-q\left(x_{g}+\alpha x_{h}\right)+r 1=0 .
$$

Let $c \in N$ such that $p_{c} \neq 0$ ( $p_{c}$ is the component of $p$ in $A_{c}$ ), then, by considering in the last equation only the component in $A_{c g h}$, we have

$$
\alpha p_{c}\left(x_{g} x_{h}+x_{h} x_{g}\right)-q_{c h} x_{g}-\alpha q_{c g} x_{h}+r_{c g h} 1=0 .
$$

But, since $g h \notin N, c g h \notin N$ and $r_{c g h}=0$. Since $g \notin N, c g \notin N$ and $q_{c g}=0$, and since $h \notin N, c h \notin N$ and $q_{c h}=0$. So the last equation shows that $x_{g} x_{h}+x_{h} x_{g}=0$, as required.

If the characteristic is equal to 3 and $\left[x_{g}, A\right] \neq 0$ or $\left[x_{h}, A\right] \neq 0$, then the argument above is valid, thus obtaining $x_{g} x_{h}+x_{h} x_{g}=0$. Otherwise, we may assume that $\left[x_{g}, A\right]=\left[x_{h}, A\right]=0$ and $x_{g} \neq 0 \neq x_{h} . A$ is not commutative (by the argument in the proof of Proposition 3.5), so there are homogeneous elements $x_{a} \in A_{a}, x_{b} \in$ $A_{b}$ with $\left[x_{a}, x_{b}\right] \neq 0$, which implies (again as in the proof of Proposition 3.5) that $\left[x_{g} x_{a}, x_{b}\right] \neq 0$ and $\left[x_{h} x_{a}, x_{b}\right] \neq 0$. Then there is a nonzero scalar $\alpha \in k$ such that $\left[\left(x_{g}+\alpha x_{h}\right) x_{a}, x_{b}\right]^{4} \neq 0$ and, as before, there are elements $p \neq 0, q, r \in N(A)$ such that $p\left(\left(x_{g}+\alpha x_{h}\right) x_{a}\right)^{2}-q\left(x_{g}+\alpha x_{h}\right) x_{a}+r 1=0$. But the algebra generated by the elements $x_{g}+\alpha x_{h}$ and $x_{a}$ is associative and commutative, so $\left(\left(x_{g}+\alpha x_{h}\right) x_{a}\right)^{2}=$ $\left(\left(x_{g}+\alpha x_{h}\right)^{2} x_{a}\right) x_{a}$. Let $c \in N$ with $p_{c} \neq 0$. Then

$$
\alpha p_{c}\left(\left(x_{g} x_{h}+x_{h} x_{g}\right) x_{a}\right) x_{a}-q_{c a h} x_{g} x_{a}-\alpha q_{c a g} x_{h} x_{a}+r_{c a^{2} g h} 1=0 .
$$

Since $g h \notin N, a^{2} \in N$ and $c \in N, c a^{2} g h \notin N$ and so $r_{c a^{2} g h} 1=0$. But ah $\notin N$ because $\left[x_{h} x_{a}, x_{b}\right] \neq 0$, which implies that $c a h \notin N$ and $q_{c a h}=0$. In the same way, $q_{\text {cag }}=0$. Hence $x_{g} x_{h}+x_{h} x_{g}=0$ and $0=\left[x_{g}, x_{h}\right]=2 x_{g} x_{h}$, a contradiction.

It remains to check that, in characteristic $3, K(A)=N(A)$. If this were not the case, there would exist an element $g \in G \backslash N$ with $\left[A_{g}, A\right]=0$. If there were another element $h \in G \backslash N$ with $g h \notin N$, we would arrive again at the contradiction $x_{g} x_{h}+x_{h} x_{g}=0$ and $0=\left[x_{g}, x_{h}\right]=2 x_{g} x_{h} \neq 0$. Therefore, $G=N \cup N g$. But $\left[A_{g}, A\right]=0$ implies that $\left[A_{a} A_{g}, A\right]=0$ for any $a \in N$ and $A$ would be commutative, a contradiction again.

Corollary 3.4 shows how $\phi$ induces a cocycle $\widehat{\phi}: G / N \times G / N \times G / N \rightarrow k^{\times}$, defined by $\widehat{\phi}(a N, b N, c N)=\phi(a, b, c)$. By the Generalised Theorem of Artin [8, Exercise 1, 
p. 40], if the associator of three elements in an alternative algebra vanishes, then the subalgebra generated by these elements is associative. In our situation, this implies that if $\left(x_{a}, x_{b}, x_{c}\right)=0$ for nonzero homogeneous elements $x_{a} \in A_{a}, x_{b} \in A_{b}$ and $x_{c} \in A_{c}$, then $\phi(H, H, H)=1$ for $H$ the subgroup generated by $a, b, c$.

Denote by $\widehat{G}$ the quotient group $G / N$ and by $\widehat{a}$ the coset $a N$ of an element $a \in G$. Given a subset $S$ of a group, $\langle S\rangle$ denotes the subgroup generated by $S$.

Proposition 3.9. For any elements $a, b, c \in G$ and $x_{a} \in A_{a}, x_{b} \in A_{b}$ :

(i) $\phi(a, b, c)=1$ if and only if the subgroup $\langle\widehat{a}, \widehat{b}, \widehat{c}\rangle$ of $\widehat{G}$ generated by $\widehat{a}, \widehat{b}, \widehat{c}$ has order at most 4 . In the other case, that is, if the order of the subgroup generated by $\widehat{a}, \widehat{b}, \widehat{c}$ is 8 , then $\phi(a, b, c)=-1$.

(ii) $x_{a} x_{b}=x_{b} x_{a}$ if $|\langle\widehat{a}, \widehat{b}\rangle| \leq 2$. For the remaining cases, $x_{a} x_{b}=-x_{b} x_{a}$.

ProOF: If $|\langle\widehat{a}, \widehat{b}, \widehat{c}\rangle| \leq 4$, then either at least two of the elements $\widehat{a}, \widehat{b}, \widehat{c}$ are equal or at least one of the elements $a, b, c$ is in $N$ or none is in $N$ but $\widehat{c}=\widehat{a} \widehat{b}=\widehat{a} \hat{b}$. In the first two cases it is clear that $\phi(a, b, c)=1$ and in the third case there exists an element $g \in N$ with $c=a b g$ and then by Lemma 2.1 and Artin's Theorem: $\phi(a, b, c)=$ $\phi(a, b,(a b) g)=\phi(a, b, a b)=1$.

If $|\langle\widehat{a}, \widehat{b}, \widehat{c}\rangle|=8$ and $0 \neq x_{a} \in A_{a}, 0 \neq x_{b} \in A_{b}$ and $0 \neq x_{c} \in A_{c}$, then by Proposition 3.8,

$$
\begin{aligned}
x_{c}\left(x_{a} x_{b}\right) & =-\left(x_{a} x_{b}\right) x_{c}=-\phi(a, b, c) x_{a}\left(x_{b} x_{c}\right) \\
& =\phi(a, b, c) x_{a}\left(x_{c} x_{b}\right)=-\phi(a, b, c) x_{c}\left(x_{a} x_{b}\right)
\end{aligned}
$$

(because $x_{a}\left(x_{c} x_{b}\right)+x_{c}\left(x_{a} x_{b}\right)=\left(x_{a} x_{c}+x_{c} x_{a}\right) x_{b}=0$ by the alternative law). Therefore $\phi(a, b, c)=-1$, and this completes the proof of part (i).

If $|\langle\widehat{a}, \widehat{b}\rangle|=4$, part (ii) follows from Proposition 3.8; while if $|\langle\widehat{a}, \widehat{b}\rangle|=2$ the result follows from Proposition 3.6.(i).

We are ready to prove the structure of the quotient group $G / N$.

THEOREM 3.10. Let $A=\bigoplus_{g \in G} A_{g}$ be a strictly alternative division quasialgebra. Then $G / N \cong \mathbb{Z}_{2} \times \mathbb{Z}_{2} \times \mathbb{Z}_{2}$.

Proof: By Proposition 3.5, any nontrivial element of $G / N$ has order 2. Besides, if $|G / N| \leq 4$, by Proposition 3.9.(i) $\phi$ is trivial and hence $A$ is associative, a contradiction. But if $|G / N|>8$, then there are elements $a_{1}, a_{2}, a_{3}, a_{4} \in G$ such that $\left|\left\langle\widehat{a}_{1}, \widehat{a}_{2}, \widehat{a}_{3}, \widehat{a}_{4}\right\rangle\right|$ $=16$. Take nonzero elements $x_{a_{i}} \in A_{a_{i}}, i=1,2,3,4$. Several applications of Proposition 3.9.(i) give:

$$
\left(x_{a_{1}} x_{a_{2}}\right)\left(x_{a_{3}} x_{a_{4}}\right)=-x_{a_{1}}\left(x_{a_{2}}\left(x_{a_{3}} x_{a_{4}}\right)\right)=x_{a_{1}}\left(\left(x_{a_{2}} x_{a_{3}}\right) x_{a_{4}}\right),
$$

but also

$$
\left(x_{a_{1}} x_{a_{2}}\right)\left(x_{a_{3}} x_{a_{4}}\right)=-\left(\left(x_{a_{1}} x_{a_{2}}\right) x_{a_{3}}\right) x_{a_{4}}=\left(x_{a_{1}}\left(x_{a_{2}} x_{a_{3}}\right)\right) x_{a_{4}}=-x_{a_{1}}\left(\left(x_{a_{2}} x_{a_{3}}\right) x_{a_{4}}\right),
$$


so that (the characteristic is not 2) $\left(x_{a_{1}} x_{a_{2}}\right)\left(x_{a_{3}} x_{a_{4}}\right)=0$, a contradiction. Therefore $G / N$ has order 8 and the result follows.

In order to describe the strictly alternative division quasialgebras, a sort of "graded Cayley-Dickson doubling process" has to be used. Let us recall the classical CayleyDickson doubling process (see $[8$, Chapter 2]) in a way suitable for our purposes.

Let $R$ be a unital associative and commutative ring and let $A$ be a unital algebra over $R$, with unit element 1 , endowed with an involution $x \mapsto \bar{x}$ satisfying $x+\bar{x} \in R 1$ and $x \bar{x}(=\bar{x} x) \in R 1$. Then we can double $A$ in the following way: Let $\alpha$ be an invertible element of $R$ and $(A, \alpha)=A \oplus A u$, (direct sum of two copies of $A$ ) with multiplication given by the multiplication in $A$ (so that $A$ becomes a subalgebra of $(A, \alpha)$ ) and by

$$
\left\{\begin{aligned}
a(b u) & =(b a) u \\
(a u) b & =(a \bar{b}) u \\
(a u)(b u) & =\alpha \bar{b} a
\end{aligned}\right.
$$

for any $a, b \in A$. The involution in $A$ is extended to an involution of $(A, \alpha)$ by means of $\overline{a u}=-a u$, for any $a \in A$. As in [7, p. 46], it follows that $(A, \alpha)$ is associative if and only if $A$ is associative and commutative, and $(A, \alpha)$ is alternative if and only if $A$ is associative.

Therefore, if the characteristic is not equal to 2 and we start with $A_{0}=R$ and the involution equal to the identity, given three invertible elements $\alpha_{1}, \alpha_{2}, \alpha_{3} \in R$, we can form the new algebras $A_{1}=\left(A_{0}, \alpha_{1}\right) \cong R[x] /\left(x^{2}-\alpha_{1}\right)$, which is commutative and associative, $A_{2}=\left(A_{1}, \alpha_{2}\right)$, which is associative but not commutative and $A_{3}=$ $\left(A_{2}, \alpha_{3}\right)$, which is strictly alternative.

In our situation, $A=\bigoplus_{g \in G} A_{g}, G / N \cong \mathbb{Z}_{2} \times \mathbb{Z}_{2} \times \mathbb{Z}_{2}$. Let $g_{1}, g_{2}, g_{3} \in G$ with $G / N=\left\langle\widehat{g}_{1}, \widehat{g}_{2}, \widehat{g}_{3}\right\rangle$ and consider the subalgebra $A_{0}=N(A)=\bigoplus_{g \in N} A_{g}$. Consider the following subgroups and subalgebras: $N_{1}=\left\langle N, g_{1}\right\rangle$ and $A_{1}=\bigoplus_{g \in N_{1}} A_{g}, N_{2}=$ $\left\langle N, g_{1}, g_{2}\right\rangle=\left\langle N_{1}, g_{2}\right\rangle$ and $A_{2}=\bigoplus_{g \in N_{2}} A_{g}$, and $N_{3}=G$ and $A_{3}=A$. Let $0 \neq x_{g_{i}} \in A_{g_{i}}$, ( $i=1,2,3)$. With $R=A_{0}$, the element $\alpha_{i}=x_{g_{i}}^{2}$ is an invertible element in $A_{g_{i}}^{2} \subseteq R$ $\left(g_{i}^{2} \in N\right)$.

Under these conditions:

PRoposition 3.11. $A_{1} \cong\left(A_{0}, \alpha_{1}\right), A_{2} \cong\left(A_{1}, \alpha_{2}\right)$ and $A=A_{3} \cong\left(A_{2}, \alpha_{3}\right)$.

Proof: Let us check, for instance, the last assertion. First, $A=A_{2} \oplus A_{2} x_{g_{3}}$ since $G=\left\langle N_{2}, g_{3}\right\rangle$. Now, for any $a, b \in N_{2}$ and $x_{a} \in A_{a}, x_{b} \in A_{b}$ :

$$
x_{a}\left(x_{b} x_{g_{3}}\right)=\frac{1}{\phi\left(a, b, g_{3}\right)}\left(x_{a} x_{b}\right) x_{g_{3}}=\left(x_{b} x_{a}\right) x_{g_{3}},
$$


because, by Proposition 3.9, if $|\langle\bar{a}, \bar{b}\rangle|=4$, then $\phi\left(a, b, g_{3}\right)=-1$ and $x_{a} x_{b}=-x_{b} x_{a}$, while if $|\langle\bar{a}, \bar{b}\rangle| \leq 2$, then $\phi\left(a, b, g_{3}\right)=1$ and $x_{a} x_{b}=x_{b} x_{a}$. Also,

$$
\left(x_{a} x_{g_{3}}\right) x_{b}=\bar{x}_{b}\left(x_{a} x_{g_{3}}\right),
$$

with $\bar{x}_{c}=x_{c}$ if $c \in N$ and $\bar{x}_{c}=-x_{c}$ if $c \notin N$, because $x_{a} x_{g_{3}}$ commutes with $x_{b}$ if $b \in N$ and anticommutes if $b \notin N$ by Proposition 3.9. Finally,

$$
\begin{aligned}
\left(x_{a} x_{g_{3}}\right)\left(x_{b} x_{g_{3}}\right) & =\frac{1}{\phi\left(a g_{3}, b, g_{3}\right)}\left(\left(x_{a} x_{g_{3}}\right) x_{b}\right) x_{g_{3}} \\
& =\phi\left(a, g_{3}, b\right)\left(\left(x_{a} \bar{x}_{b}\right) x_{g_{3}}\right) x_{g_{3}} \\
& =\left(\phi\left(a, g_{3}, b\right) x_{a} \bar{x}_{b}\right)\left(x_{g_{3}}\right)^{2} \\
& =\left(\bar{x}_{b} x_{a}\right) \alpha_{3} .
\end{aligned}
$$

Let us denote by $K$ the field $A_{e}$, then $\operatorname{dim}_{K} A_{g}=1$ for any $g \in G$ and hence the quasialgebra $A$ may be identified with $K_{F} G$ for a suitable 2-cochain $F: G \times G \rightarrow K^{\times}$. If $F_{0}$ denotes the restriction of $F$ to $N \times N$, since $N(A)=A_{0}=\bigoplus_{g \in N} A_{g}$ is commutative, $F_{0}: N \times N \rightarrow K^{\times}$is symmetric $\left(F_{0}(a, b)=F_{0}(b, a)\right.$ for any $\left.a, b \in N\right)$ and since $N(A)$ is associative, $F_{0}$ is a 2 -cocycle.

The Cayley-Dickson process can be reinterpreted in this situation, thus extending [2, Proposition 4.1]:

Definition: Let $K$ be a field, $G$ an Abelian group and $N \leq S \leq T \leq G$ a chain of subgroups with $[T: S]=2$ and $T=S \cup S g$ with $g^{2} \in N$. Let $\beta: G \mapsto K$ be the map given by $\beta(g)=1$ if $g \in N$ and $\beta(g)=-1$ if $g \notin N$. Let $F: S \times S \rightarrow K^{\times}$be a 2 -cochain such that

$$
\frac{F\left(s_{1}, s_{2}\right)}{F\left(s_{2}, s_{1}\right)}=\beta\left(s_{1}\right) \beta\left(s_{2}\right) \beta\left(s_{1} s_{2}\right)\left(=(\partial \beta)\left(s_{1}, s_{2}\right)\right)
$$

for any $s_{1}, s_{2} \in S$ and let $0 \neq \alpha \in K$.

Then the 2-cochain $\bar{F}=F_{(r, g, \alpha)}: T \times T \rightarrow K^{\times}$defined by

$$
\begin{aligned}
\bar{F}(x, y) & =F(x, y) \\
\bar{F}(x, y g) & =F(y, x) \\
\bar{F}(x g, y) & =\beta(y) F(x, y) \\
\bar{F}(x g, y g) & =\beta(y) F(y, x) \alpha
\end{aligned}
$$

for any $x, y \in S$, is said to be the 2 -cochain extending $F$ by means of $(T, g, \alpha)$.

It is easy to check that the new 2 -cochain $\bar{F}$ above still satisfies

$$
\frac{\bar{F}\left(t_{1}, t_{2}\right)}{\bar{F}\left(t_{2}, t_{1}\right)}=(\partial \beta)\left(t_{1}, t_{2}\right)
$$


for any $t_{1}, t_{2} \in T$.

The next result summarises most of the work done in this section and provides the characterisation of strictly alternative division quasialgebras.

THEOREM 3.12. Let $k$ be a field of characteristic not equal to 2 and let $K / k$ be a field extension. Let $G$ be an Abelian group, $N$ a subgroup of $G$ such that $G / N \cong \mathbb{Z}_{2} \times \mathbb{Z}_{2} \times \mathbb{Z}_{2}$ and $N_{1}$ and $N_{2}$ subgroups of $G$ such that $N<N_{1}<N_{2}<G$. Let $\beta: G \mapsto K^{\times}$be the map given by $\beta(x)=1$ for $x \in N$ and $\beta(x)=-1$ for $x \notin N$, and $F_{0}: N \times N \mapsto K^{\times}$a symmetric 2-cocycle. Let $g_{1}, g_{2}, g_{3} \in G$ with $N_{1}=\left\langle N, g_{1}\right\rangle, N_{2}=\left\langle N_{1}, g_{2}\right\rangle$ and $G=\left\langle N_{2}, g_{3}\right\rangle$, and let $\alpha_{1}, \alpha_{2}, \alpha_{3}$ be nonzero elements in $K$. Consider the extended 2-cochains $F_{1}=\left(F_{0}\right)_{\left(N_{1}, g_{1}, \alpha_{1}\right)}, F_{2}=\left(F_{1}\right)_{\left(N_{2}, g_{2}, \alpha_{2}\right)}$ and $F_{3}=\left(F_{2}\right)_{\left(G, g_{3}, \alpha_{3}\right)}$. Then $K_{F_{3}} G$ is a strictly alternative division quasialgebra over $k$.

Conversely, if $k$ is a field and $A$ is a strictly alternative division quasialgebra over $k$ then the characteristic of $k$ is not equal to 2 and there are $K, G, N, F_{0}, \alpha_{1}, \alpha_{2}, \alpha_{3}$ satisfying the preceding conditions such that $A \cong K_{F_{3}} G$.

In particular, this result shows that any strictly alternative division quasialgebra is obtained as a "twisting" $K_{F} G$ of a group algebra (over a field extension of the ground field) of an Abelian group.

In case $A=\bigoplus_{g \in G} A_{g}$ is a strictly alternative division quasialgebra over the field $k$ and there is a subgroup $\bar{N}$ of $G$ such that $G=N \times \bar{N}$, then $\bar{N} \cong \mathbb{Z}_{2} \times \mathbb{Z}_{2} \times \mathbb{Z}_{2}$ and it can be immediately concluded that $A$ is the tensor product (over $K=A_{e}$ ) of the commutative and associative division quasialgebra $N(A)=A_{N}=\bigoplus_{g \in N} A_{g}$ and the strictly alternative division quasialgebra $A_{\bar{N}}=\bigoplus_{g \in \bar{N}} A_{g}$ which is a Cayley-Dickson algebra over $K$ (it is obtained by applying the Cayley-Dickson doubling process three times over $K$ ).

\section{REFERENCES}

[1] H. Albuquerque, A. Elduque and J.M. Pérez-Izquierdo, ' $\mathbb{Z}_{2}$-quasialgebras' (to appear).

[2] H. Albuquerque and S. Majid, 'Quasialgebra Structure of the Octonions', J. Algebra 200 (1999), 188-224.

[3] H. Albuquerque and S. Majid, ' $\mathbb{Z}_{n}$-Quasialgebras', in Matrices and Group Representations, (A.P. Santana, A.L. Duarte and J.F. Queiro, Editors), Textos de Mat. B 19 (Universidade de Coimbra, Coimbra, 1999), pp. 57-64.

[4] S. Berman, Y. Gao, Y. Krylyuk and E. Neher, 'The alternative torus and the structure of elliptic quasi-simple Lie algebras of type $A_{2}$ ', Trans. Amer. Math. Soc. 347 (1995), 4315-4363.

[5] C. Năstãsescu and F. Van Oystaeyen, Graded ring theory (North-Holland, Amsterdam, 1982). 
[6] J.M. Osborn and D.S. Passman, 'Derivations of skew polynomial rings', J. Algebra 176 (1995), 417-448.

[7] R.D. Schafer, An introduction to nonassociative algebras (Academic Press, New York, 1966).

[8] K.A. Zhevlakov, A.M. Slin'ko, I.P. Shestakov and A.I. Shirshov, Rings that are nearly associative (Academic Press, New York, 1982).

Departamento de Matematica

Faculdade de Ciencias e Tecnologia

Universidade de Coimbra

3000 Coimbra

Portugal

Departamento de Matemáticas y Computación

Universidad de La Rioja

26004 Logroño

Spain
Departamento de Matemáticas

Facultad de Ciencias

Universidad de Zaragoza

50009 Zaragoza

Spain 